archives-ouvertes

\title{
Introducing spatial neighbourhood in Evidential C-Means for segmentation of multi-source images: Application to prostate multi-parametric MRI
}

Nasr Makni, Nacim Betrouni, Olivier Colot

\section{- To cite this version:}

Nasr Makni, Nacim Betrouni, Olivier Colot. Introducing spatial neighbourhood in Evidential C-Means for segmentation of multi-source images: Application to prostate multi-parametric MRI . 2014. hal01183343

\section{HAL Id: hal-01183343 \\ https://hal.archives-ouvertes.fr/hal-01183343}

Submitted on 2 Sep 2015

HAL is a multi-disciplinary open access archive for the deposit and dissemination of scientific research documents, whether they are published or not. The documents may come from teaching and research institutions in France or abroad, or from public or private research centers.
L'archive ouverte pluridisciplinaire HAL, est destinée au dépôt et à la diffusion de documents scientifiques de niveau recherche, publiés ou non, émanant des établissements d'enseignement et de recherche français ou étrangers, des laboratoires publics ou privés. 


\title{
Introducing spatial neighbourhood in Evidential C-Means for segmentation of multi-source images : application to prostate multi-parametric MRI
}

\author{
Nasr Makni ${ }^{\mathrm{a}}$, Nacim Betrouni ${ }^{\mathrm{a}, \mathrm{d}}$, Olivier Colot ${ }^{\mathrm{b}, \mathrm{c}}$ \\ ${ }^{a}$ INSERM U703, F-59000 Lille, France \\ ${ }^{b}$ Univ Lille Nord de France, F-59000 Lille, France \\ ${ }^{c}$ LAGIS FRE 3303, Villeneuve d'Ascq 59650, France \\ ${ }^{d}$ CHULille, F-59000 Lille, France
}

\begin{abstract}
In this paper we introduce an evidential multi-source segmentation scheme for the extraction of prostate zonal anatomy using multi-parametric MRI. The Evidential C-Means (ECM) classifier was adapted to a segmentation scheme by introducing spatial neighbourhood-based relaxation step in its optimisation process. In order to do so, basic belief assignments on voxels membership were relaxed using distance-weighted combination of belief from spatial neighbours. For the application on prostate tissues, geometric $a$ priori was modelled and used as an additional data source. Our method was first experimented on simulated images to prove the improvement brought to the ECM. A validation study of the segmentation method was then conducted on 31 patients MRI data. Each MRI was manually segmented by three independent expert radiologists, and an estimated truth was computed using STAPLE algorithm, for inter-observer variability was taken into account. This validation proved that segmentation obtained with our method is accurate and comparable to expert segmentation. We also show that our segmentation scheme enables to detect and highlight outliers, which could be interpreted by physicians as irregular tissues. The use of belief functions also provides additional information on borders between structures. We do believe these are sources of evidence that could help physicians/algorithms in characterising tissues and structures.
\end{abstract}

\footnotetext{
${ }^{2}$ Courtesy of P. Puech and A. Iancu expert radiologists from University Hospital of Lille for contributing in this study.

${ }^{*}$ Corresponding author

**Principal corresponding author
} 
Keywords: belief functions, Evidential C-Means, Dempster-Shafer theory, Segmentation, prostate, zonal anatomy, multi-parametric MRI, peripheral zone, transition zone

\section{Introduction}

It is known that acquired data is imprecise, uncertain, incomplete, and distorted by sensors or observers it emanates from. In such a context, the use of multi-sensor data sources brings an important enhancement in data 5 analysis as different sensors have their own physical characteristics, and consequently do not necessarily detect the same features of the real scene. This statement is specially relevant in the field of medical image analysis where the use of different imaging modalities and/or acquisition techniques allows having complementary information on in vivo tissues, pathologies, etc. In many cases, physicians tend to use "multi-sensor" images to refine reliability of their image-based diagnosis. We shall distinguish two types of data implied by multi-sensor. The first one is data provided by different imaging modalities. The second one is provided by a single imaging modality, using several acquisition techniques/parameters making the imaging device act like a different sensor. In the context of MR Imaging, physicians use the terms "multi-parametric", "multi-modal" or "multi-spectral". In this field, there is an increasing interest in adapting image analysis techniques to multi-sensor schemes $[1,2,3,4]$. Many authors have worked on fusion and segmentation of multi-sensor images, and this technique proved to be efficient in many application fields, especially medical images analysis. Flach et al. [4] focused on registration and segmentation of multi-modal images (MRI, CT scans, Ultrasound, etc.), and proposed a generic method based on Gibbs probability distribution. Chun et al. [5] also used Gibbs-Markov random fields (GRF and MRF) for a Bayesian classification of multi-echo

25 MRI. Bricq et al. [6] proposed a unifying framework using MRF for multiecho brain MRI segmentation. Lee et al. [3] modelled multi-echo image regions using a similar approach ( $\mathrm{GRF}$ and $\mathrm{MRF}$ ), but compared results from Bayesian MAP and the evidential reasoning [7, 8], also known as belief functions theory. These authors could establish that the evidential approach 30 was less restrictive than the MAP decision. We believe multi-sensor segmentation methods should rely on models that take into account redundancy and conflicts between data sources. Evidence theory, also called DempsterShafer theory $[8,7]$, is widely used in data fusion and pattern recognition [9] as it provides strong and native modelling of imprecision, data fusion, 
eventual conflictual sources and outliers rejection [10,11, 12, 13, 14, 15]. There are several applications of evidence theory in medical images analysis and computer aided diagnosis. In [3, 16], evidential modelling was used for segmentation of brain MRI. In [17] it was used for segmentation of left ventricle of the heart. In $[2,15]$ Bloch as well as Capelle et al. introduced evidential classification of dual-echo brain MRI in a pathological context.

In the context of prostate cancer diagnosis, image-based examination routines now include both morphological studies (e.g. T2-weighted MRI) and functional imaging (e.g. diffusion weighted and T1-weighted contrastenhanced MRI). These multiple imaging studies provide a huge amount of complementary but also redundant and highly imprecise information, which is used by physicians in early detection and staging of prostate cancer. It also allows a better characterisation of prostate key substructures like the peripheral zone and the center of the gland [18]. The prostate consists of four zones : peripheral zone (PZ), central zone (CZ), transition zone (TZ) and fibro-muscular structure (figure 1). With aging, the periurethral tissue and the TZ may considerably hypertrophy, gradually compressing the CZ and stretching the PZ. This hyperplasia essentially does not involve the PZ and therefore, only two areas may be considered from a radiologic point of view : the central gland (hypertrophied transition zone and periurethral glands) and PZ. In this paper, Transition Zone (TZ) refers then to the complementary of PZ. PZ and TZ are key structures, as $80 \%$ of prostate tumours are located within PZ, and TZ/Prostate volumes' ratio can be used to monitor dysfunctions of the gland. Manually outlining these structures is a tedious task and not feasible in clinical routine and, to our knowledge, no automatic segmentation method had been proposed in literature.

In this study, we introduce a novel evidential segmentation method for classification of prostate zones on multi-parametric MRI. Our segmentation process is based on the Evidential C-Means (ECM) recently introduced by Masson and Denoeux [19]. The contributions of our method are the introduction of voxels' spatial neighbourhood information in the optimization process of the ECM, and the use of a spatial a priori, deduced from a mathematical modelling based on catenaries geometry. The paper is organised as follows: in Section 2, we briefly describe theoretical background and rational of our study. In Section 3, we detail our methods for introducing spatial neighbourhood and modelling an a priori-based feature. In Section 4, we experiment and show the improvements enabled by our contributions, and we detail the validation of our method for use in a clinical context. Section 5 concludes the paper. 


\section{Background}

\section{2.1. Belief functions theory}

Belief functions theory, also called evidential reasoning, was first introduced by Dempster in 1967 [8] and was formalised as a theory by [7]. Smets and Kennes' Transferable Belief Model (TBM) [20] brought a coherent interpretation of the underlying concept of the theory, far from the restricted probabilistic interpretation. The modelling associates a source of data - or sensor - $S$, to a frame of discernment $\Omega=\left\{\omega_{1}, \ldots, \omega_{k}\right\}$ composed of the $k$ hypotheses that can be observed by $S$. This model extracts belief on all subsets of $\Omega$, called propositions. A proposition $\left\{\omega_{i}, \ldots, \omega_{j}\right\} \in 2^{\Omega}$ is quantified by a basic belief assignment (bba). A bba $m()$ is a function from ${ }_{85} 2^{\Omega}=\left\{\emptyset, \omega_{1}, \ldots, \omega_{k}, \omega_{1} \cup \omega_{2}, \omega_{1} \cup \omega_{3}, \ldots, \Omega\right\}$ to the range $[1,0] . m()$ must satisfy the following condition:

$$
\sum_{A \subseteq \Omega} m(A)=1
$$

Let $A \subseteq \Omega$ be a proposition: the higher $m(A)$ the stronger the belief on the proposition $A$.

Several operations can be performed on bbas: discounting [21], combination [22], transformation to probability measures [23], etc. For a better readability of this paper, some of these items that are used in this work are defined in the context of the application, and thus are detailed in the Methods section.

\subsection{Evidential C-Means (ECM)}

Masson and Denoeux [19] proposed a novel C-Means classifier using belief functions and strongly inspired by the fuzzy c-means and the noise-clustering algorithm [24]. This evidential classifier, called ECM, optimises a credal partition, which could be assimilated to membership partition in Fuzzy CMeans approach.

Let us consider a set of $N$ patterns to be classified in $C$ classes: $\Omega=$ $\left\{\omega_{1}, \ldots, \omega_{C}\right\}$. For each pattern $P_{i}, 1 \leq i \leq N$, a basic belief assignment $m_{i}$ is defined on $2^{\Omega}$. A $N \times 2^{C}$ matrix $M$ is defined as the credal partition. Each row of $M$ is a bba $m_{i}, 1 \leq i \leq N$ :

$$
\begin{gathered}
\forall 1 \leq j \leq 2^{C} \\
\left\{\begin{array}{c}
\mathrm{M}_{\mathrm{ij}}=m_{i}\left(A_{j}\right) \\
A_{j} \subseteq \Omega,
\end{array}\right.
\end{gathered}
$$


The bbas $\left\{m_{1}, \ldots, m_{N}\right\}$ are derived from patterns object data (non re-

lational) based on distance measure. In addition to the $C$ classes, a noise cluster is also defined by a threshold value as introduced in [24].

ECM's optimisation process consists in looking for a credal partition $M$ and a matrix $V$ of classes centres, that both minimize the following objective function

$$
J_{E C M}(M, V) \triangleq\left[\sum_{i=1}^{N} \sum_{\substack{A_{j} \subseteq \Omega \\ A_{j} \neq \emptyset}}\left|A_{j}\right|^{a} \cdot m_{i}\left(A_{j}\right)^{b} \cdot d_{i j}^{2}\right]+\sum_{i=1}^{N} \delta^{2} \cdot m_{i}(\emptyset)^{b}
$$

where $a$ and $b$ are weighting parameters (default values, given in [19], are equal to 2 ), and $\delta$ is a threshold distance to the outliers "class". $d_{i j}$ is the distance from pattern $P_{i}$ to $V_{j}$ the "barycentre" of $A_{j}$. Indeed, using a C-Means approach demands the definition of classes' centre. In Evidential C-Means, Masson and Denoeux [19] defined barycentres, which are centres of $\Omega$ 's subsets. Let $V_{j}\left(1 \leq j \leq 2^{c}\right)$ be the $s \times 1$ column vector representing the barycentre of element $A_{j} \subseteq \Omega$. $V_{j}$ is given by

$$
V_{j}=\frac{1}{\left|A_{j}\right|} \sum_{k=1}^{C} \delta_{k j} \overline{\omega_{k}}
$$

where $\delta_{k j}$ equals 1 if $\omega_{k} \in A_{j}$ ( 0 else) and $\overline{\omega_{k}}$ is the $s \times 1$ column vector of features representing the centre of class $\omega_{k}$.

\section{Methods}

\subsection{Definitions \& assumptions}

We assume $s$ volume data sources $\left\{S_{d}\right\}_{1 \leq d \leq s}$, and $v(x, y, z)$ a voxel having $(x, y, z)$ as volume coordinates. To $v(x, y, z)$ we associate the pattern

$$
P=\left[p_{1}, \ldots, p_{s}\right]
$$

where $p_{d}, 1 \leq d \leq s$, is the intensity of $v(x, y, z)$ according to data source $d$. Let $\Omega=\left\{\omega_{1}, \ldots, \omega_{c}\right\}$ be the set of $c$ classes that represent volume regions we have to segment. $\Omega$ is also called frame of discernment. We note $2^{\Omega}=\left\{A_{j}\right\}_{1 \leq j \leq 2^{c}}$ the powerset, or set of subsets, of $\Omega$. The segmentation process we propose classifies each pattern $P$ in one of $\Omega$ 's elements, while taking into account its spatial position $(x, y, z)$ and information from its connected neighbours. These neighbours are identified using a chosen connexity system. 


\subsection{Basic Belief Assignment extraction}

The first step in this evidential segmentation process is the extraction of Basic Belief Assignment on the membership of voxels. We use the distancebased model introduced in the ECM [19]. According to this method, for each voxel $v_{i}$ a bba $m_{i}$ is defined on elements of $2^{\Omega}$ by

$$
\begin{aligned}
& \forall A_{j} \subseteq \Omega, A_{j} \neq \emptyset \\
& \left\{\begin{array}{l}
m_{i}\left(A_{j}\right)=\frac{\left|A_{j}\right|^{-a /(b-1)} \cdot d_{i j}^{-2 /(b-1)}}{\sum_{A_{f} \subseteq \Omega,}\left|A_{f}\right|^{-a /(b-1)} \cdot d_{i f}^{-2 /(b-1)}+\delta^{-2 /(b-1)}} \\
m_{i}(\emptyset)=1-\sum_{A_{j} \neq \emptyset} m_{i}\left(A_{j}\right)
\end{array}\right.
\end{aligned}
$$

where $d_{i j}$ is the distance from voxel $v_{i}$ to the barycentre of element $A_{j}$. The set of bbas $\left\{m_{i j}\right\}_{\substack{i=1 \ldots N \\ j=1 \ldots 2^{C}}}$ is then used to build $M$, be the credal partition as defined in section 2.2 (equation 2).

\subsection{Introducing spatial neighbourhood}

The ECM classifier extracts and optimizes partial knowledge on patterns' membership, and each pattern is a d-dimensional vector of the associated voxel values. In a segmentation context, this model takes no account of voxels' spatial context and treats them as spatially independent objects. It is obvious however that a voxel's neighbourhood, as defined by a connexity system, brings valuable information. Indeed, image segmentation supposes that image regions are sharing common features. Connexity systems, or neighbourhood, model this assumption in region-oriented segmentation methods, such as growing region, Markov Fields/Chains, etc.

We assume that within an image region, or class, a bba is not only a knowledge on a voxel, but also a partial knowledge on its neighbours. Corrupted information, extracted from outliers, also known as noise patterns, can then be fixed by its neighbours, which is one of the principles of noisereducing methods and filters. Thus, introducing neighbourhood information in the ECM modelling would :

- model contextual region information in extracting knowledge on patterns/voxels.

- reduce corrupted information, related to outliers and noise.

- assimilate the ECM classifier to a region-based segmentation process.

We focus on tools proposed by belief functions theory for merging and combining bbas. The algorithm we propose is then a modified ECM: MECM. 


\subsubsection{Combining neighbour bbas}

Let $m_{i}$ be the bba of pattern $P_{i}$, associated to voxel $v_{i}(x, y, z)$. We relax $m_{i}$ by combining it with bbas from spatially connected neighbours. Spatial neighbourhood is defined by an $n c$-connexity system (26-connexity,

8-connexity, 6-connexity,..). Combining these bbas is possible using one of the evidential combination rules, for which a review can be found in [22].

Let $m_{i}^{\cap}$ be the result of combining $m_{i}$ with bbas from the $(n c-1)$ connected voxels, using a non-normalised conjunctive combination [22]. $m_{i}^{\bigcap}$ is defined by

$$
\begin{aligned}
& \forall A \subseteq \Omega, \\
& m_{i}^{\cap}(A)=\sum_{A_{1} \cap \ldots \cap A_{f}=A} m_{1}\left(A_{1}\right) \times \ldots \times m_{n c}\left(A_{f}\right) \\
& A_{1}, \ldots, A_{f} \subseteq \Omega
\end{aligned}
$$

where $m_{1} \ldots m_{n c}$ are bbas from $v_{i}^{\prime}$ neighbours.

This combination rule is capable of quantifying eventual conflict between the combined bbas, which is represented by $m_{i}^{\cap}(\emptyset) \neq 0$. This information could be valuable in some applications, e.g. medical images segmentation, but is known to be hard to interpret. In a closed-world assumption context [25], where all the hypotheses on the membership of image sites are included in the frame of discernment $\Omega$, all bbas have to verify that $m_{i}^{\bigcap}(\emptyset)=0$. When using a conjunctive combination, an orthogonal rule, also called Dempsters rule of combination [10], is modelled to normalise the result of bbas' combination to fulfil the condition $m_{i}^{\bigcap}(\emptyset)=0$. Let $m_{i}^{\oplus}$ be the result of combination using the orthogonal rule, it is then defined by

$$
\begin{gathered}
\forall A \subseteq \Omega, A \neq \emptyset \\
\left\{\begin{array}{c}
m_{i}^{\oplus}(A)=\frac{m_{i}^{\cap}(A)}{1-m_{i}^{\cap}(\emptyset)} \\
m_{i}^{\oplus}(\emptyset)=0
\end{array}\right.
\end{gathered}
$$

Both conjunctive combination rules 7 and 8 can be used to introduce spatial neighbourhood information, though there are some differences that we interpret and discuss in the dedicated section of this paper.

\subsubsection{Weighting neighbour bbas}

185 Intuitively, the amount of trust in the information brought by a neighbour is higher (lower) when it is closer (further). We model this reliability of neighbour bbas using a weakening factor which is inversely proportional to the distance from the voxel. Let $m_{k}$ be a neighbour of $m_{i}$ : we define a discounting factor $0 \leq \alpha_{k} \leq 1$ such as : 


$$
\alpha_{k}=\frac{\gamma}{d_{k}^{2}}
$$

where $0 \leq \gamma \leq 1$ is a weighting parameter, and $d_{k}$ is a normalised distance (divided by the least distance) between $v_{i}$ and its spatial neighbour $v_{k}$. The optimal value of $\gamma$ can be estimated by experiments/cross validation on images with well-known ground truth.

Let $m_{k}^{\alpha_{k}}$ the $\alpha_{k}$-discounted bba :

$$
\begin{aligned}
& \forall A \subseteq \Omega \\
& \left\{\begin{array}{l}
m_{k}^{\alpha_{k}}(A)=\alpha_{k} \cdot m_{k}(A) \\
m_{k}^{\alpha_{k}}(\Omega)=1-\alpha_{k}+\alpha_{k} \cdot m_{k}(\Omega)
\end{array}\right.
\end{aligned}
$$

A distance-weighted belief combination is then possible using discounted bbas $m_{1}^{\alpha_{1}}, \ldots, m_{n c}^{\alpha_{n c}}$ :

$$
m_{i}^{\odot}=\underset{k=1 \ldots n c}{\odot} m_{k}^{\alpha_{k}}
$$

where $\odot$ is one of the conjunctive operators, $\cap$ and $\oplus$, defined in 7 and 8 , respectively.

This distance-based discounting is particularly relevant in the case of some 3D imaging techniques (e.g. MRI end PET scan in medical imaging) where voxels are anisotropic. Indeed, these techniques use a set of parallel slices to reconstruct a 3D volume, and slices' thickness and/or distance can be different from pixels' size, which causes partial volume effect. For instance, in our application, $1.5 \mathrm{~T}$ prostate morphological T2-weighted MRI, used in clinical routine in our radiology department, has $0.86 \times 0.86 \times 4.0 \mathrm{~mm}^{3}$ sized voxels. This discounting is also relevant when using a multi-scale approach, keeping the influence of neighbours moderated by their distance even when using rougher scales.

\subsubsection{Credal relaxation}

Based on the modelling of bbas extraction and correction presented above, we propose to modify the ECM algorithm by adding a relaxation step within its iterative process. In Masson and Denoeux's ECM, the latter consists, in each loop, in extracting the credal partition $M$ using (2) and optimise classes' centres $V$ using a linear resolution of the objective function minimisation (3). The minimisation problem is resolved in [19] as the resolution of the following linear system :

$$
H V=B
$$


where

$$
\left\{\begin{array}{l}
H_{l k}=\sum_{i} \sum_{A_{j} \supseteq\left\{\omega_{l}, \omega_{k}\right\}}\left|A_{j}\right|^{a-1} \cdot m_{i}^{b}\left(A_{j}\right) \\
B_{l q}=\sum_{i} x_{i q} \sum_{\omega_{l} \in A_{j}}\left|A_{j}\right|^{a-1} \cdot m_{i}^{b}\left(A_{j}\right)
\end{array}\right.
$$

In our scheme, we introduce an additional step in computing the credal partition, which we call a relaxation step. Indeed, after extracting voxels' bbas as independent patterns using (6), we combine each bba with its spatially connected bbas using (11). The new algorithm is then detailed in the following section.

\begin{tabular}{ll} 
Input & $n$ patterns $\left\{\left[P_{1}^{i}, \ldots, P_{s}^{i}\right]\right\}_{1 \leq i \leq n}$ \\
\hline Parameters & $a>0:$ weighting exponent (default value 2) \\
& $b>0:$ weighting exponent (default value 2$)$ \\
& $\delta>0:$ rejection factor \\
& $0 \leq \gamma \leq 1:$ neighbourhood weakening parameter \\
& $\varepsilon>0:$ convergence threshold \\
\hline Initialisation & Initialize $V_{0} ;$ \\
\hline Loop & $t \leftarrow 0$ \\
& $t \leftarrow t+1 ;$ \\
& Compute $M_{t}$ using $(2),(6)$ and $V_{t-1} ;$ \\
& Compute the relaxed $M_{t}^{\odot}$ using $(11) ;$ \\
& Compute the new $V_{t}$ using $M_{t}^{\odot}$ and $(12) ;$ \\
& Until $\left\|V_{t}-V_{t-1}\right\|<\varepsilon$ \\
\hline
\end{tabular}

Table 1: MECM: Modified ECM algorithm for neighbourhood-based credal relaxation.

\subsubsection{Decision and labelling}

At the level of the algorithm described in table 1, bbas on membership of voxels are extracted and optimised in a connexity-aware Evidential CMeans scheme. This level, called the credal level in Smets' Transferable Belief Model [20], is dedicated to knowledge extraction and combination. We do believe and show, in the discussion section of this paper, that this level of knowledge may provide valuable information to physicians, which could help them in characterising complex tissues' mixture and detecting abnormal structures/lesions. Nevertheless, we still have to make a decision on voxels' membership to perform a segmentation. Decision making, or labelling, can be reached by transforming bbas into probability measures. We use the pignistic transformation [23], in which the amount of belief 
assigned to the empty set $\emptyset$ and $\Omega$ 's subsets that include more than one class are equally redistributed to the singletons (classes/elements of $\Omega$ ). A probability function, called pignistic probability, is then computed using

$$
\begin{aligned}
& \forall \omega \in \Omega, \\
& \operatorname{BetP}(\omega) \triangleq \frac{1}{1-m(\emptyset)} \sum_{\omega \in A} \frac{m(A)}{|A|}
\end{aligned}
$$

\section{Application to segmentation of prostate zones}

\subsection{Assumptions}

We apply the proposed segmentation method to segment prostate MRI into Peripheral Zone (PZ) and its complementary (TZ) (c.f. Introduction). We then assume $\Omega=\{P Z ; T Z\}$. Multi-parametric MR volumes are used for data sources: T2-weighted, Diffusion-weighted and T1-weighted Contrast45 Enhanced MRI are used. We assume prostate contours had been delineated on these images, using our automatic segmentation method [26] and, if needed, expert manual correction. The region of interest of our segmentation process is then limited to prostate voxels.

\subsection{Modelling an a priori feature}

250 If multi-parametric MRI enhance prostate's tissue characterisation, some structures may have similar intensities and the corresponding voxels may have very similar features. The most known illustration of this issue is the overlap of intensities from the peripheral zone with those from cysts of the transition zone (figure 1). Physicians are able to distinguish these structures based on their location within the prostate. One of the fundamentals of this a priori anatomy is that $\mathrm{TZ}$ is surrounded by the $\mathrm{PZ}$ which is located on the posterior face of the prostate (figure 1). Indeed, relative positions of $\mathrm{PZ}$ and $\mathrm{TZ}$ are invariant, as the TZ holds the centre of the gland, and PZ is situated in posterior and lateral positions.

We propose to model this information as an additional a priori feature which brings information on voxels' localisation inside the gland, and regarding the relative positions of $\mathrm{TZ}$ and $\mathrm{PZ}$. Intuitively, the aspect of the $\mathrm{PZ}$ on axial MRI slices (figure 1) may be modelled by catenaries-like curves. The catenary, or funicular curve, is the theoretical shape of a hanging flexible chain or cable when supported at its ends and acted upon by its own weight 


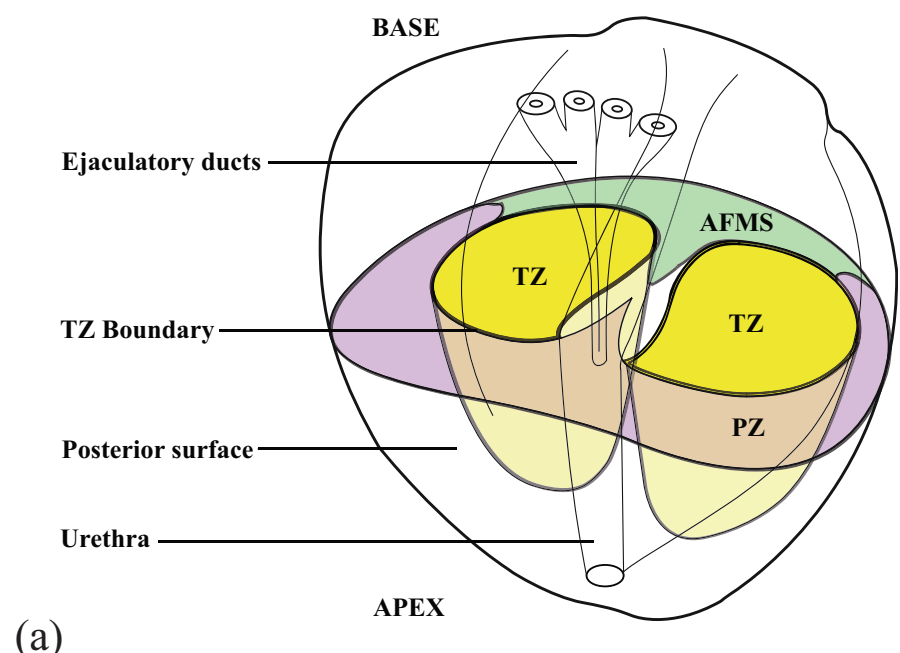

(a)

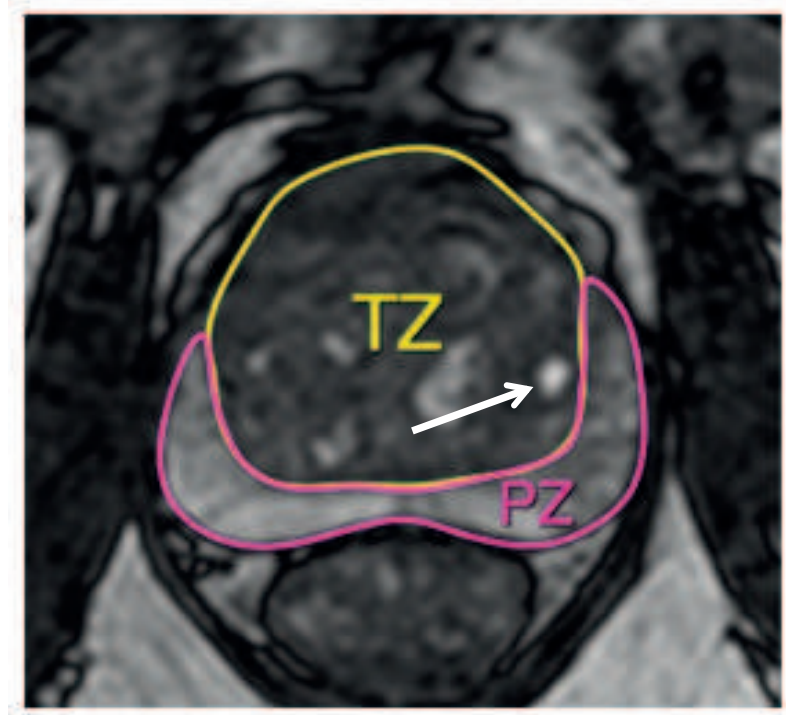

(b)

Figure 1: anatomic description (a) and T2-weighted MRI (b) of an average 60cc prostate showing its zonal anatomy with a transverse section at the verumontanum level (TZ: Transition zone, PZ: peripheral zone, AFMS: anterior fibromuscular stroma). The white arrow in (b) shows a cyst in the $\mathrm{TZ}$ that has intensities that are very similar to $\mathrm{PZ}$. 
and in equilibrium. This shape is similar in appearance to a parabola, and its model in Cartesian coordinates is given by:

$$
y=\theta \cdot \cosh \left(\frac{x}{\theta}\right)+c s t=\frac{\theta}{2}\left(e^{x / \theta}+e^{-x / \theta}\right)+c s t
$$

Where $(x, y)$ are planar coordinates, $\theta$ is the catenary's parameter that characterises its curvature, and cst a constant that simply translates the curve. Catenary curves are given in figure 2.a, for different values of $\theta$.

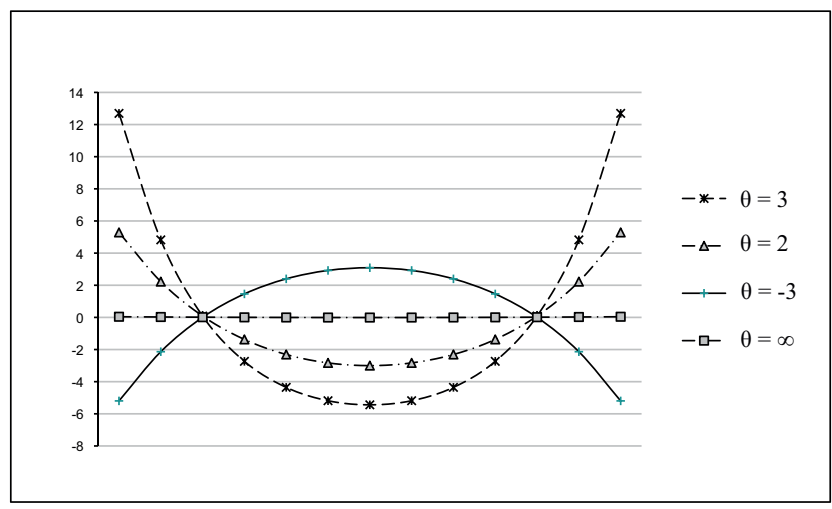

(a)

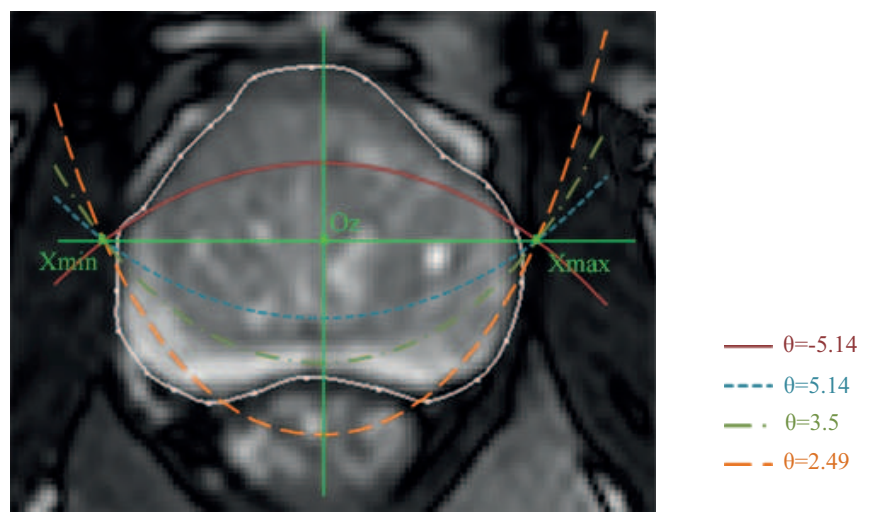

(b)

Figure 2: (a) catenary curves ; (b) catenaries on prostate MRI (axial view)

Let us consider the $z$ th axial plan, or "slice", of prostate MRI. Assuming the gland pre-delineated, let $G^{z}\left(G_{x}, G_{y}\right)$ be the gravity center of prostate voxels from the $z$ th axial slice. Let $\left(O_{z}, \vec{x},-\vec{y}\right)$ be a "gravity" Cartesian 
coordinate system such as

$$
O_{z}=\left(\frac{\mathrm{x}_{\min }^{z}+\mathrm{x}_{\max }^{z}}{2} ; \mathrm{G}_{\mathrm{y}}\right)
$$

275 where $x_{\min }^{z}$ and $x_{\max }^{z}$ are minimal and maximal x coordinates of prostate voxels in axial plan $z$, respectively. $x_{\min }^{z}$ and $x_{\max }^{z}$ can be easily computed by searching for minimal and maximal $\mathrm{x}$-coordinates among prostate pixels of the $z$ th slice. For each prostate voxel $\mathrm{V}_{i}(\mathrm{x}, \mathrm{y}, \mathrm{z})$, there is one, and only one, catenary $\mathrm{Cat}_{\theta_{i}, \text { cst }_{i}}$ that is suspended from endpoints $X_{\min }$ and $X_{\max }$ ${ }_{280}$ (figure 2.b), where the parametric catenary function $C_{a t} t_{\theta, c s}$ is defined by

$$
\begin{aligned}
& \text { Cat }_{\theta, c s t}: R \rightarrow R \\
& x \mapsto \theta \cdot \cosh \left(\frac{x}{\theta}\right)+c s t
\end{aligned}
$$
by

We then compute the catenary-based features associated to voxels $\left\{v_{i}\left(x_{i}, y_{i}, z_{i}\right)\right\}_{1 \leq i \leq n}$,

$$
\begin{aligned}
& \text { Cat }: R^{3} \rightarrow R \\
& (x, y, z) \mapsto\left\{\begin{array}{l}
+\frac{1}{\theta^{2}} \text { if } y \leq G_{y} \\
-\frac{1}{\theta^{2}} \text { else }
\end{array}\right.
\end{aligned}
$$

where $G_{y}$ is the $y$ coordinate of the gravity centre $G^{z}\left(G_{x}, G_{y}\right)$.

Cat enables then to compute, for each voxel, a new feature, a new feature 285 based on its location within the gland. Figure 3) shows how Cat-based features is used as an additional data source. 


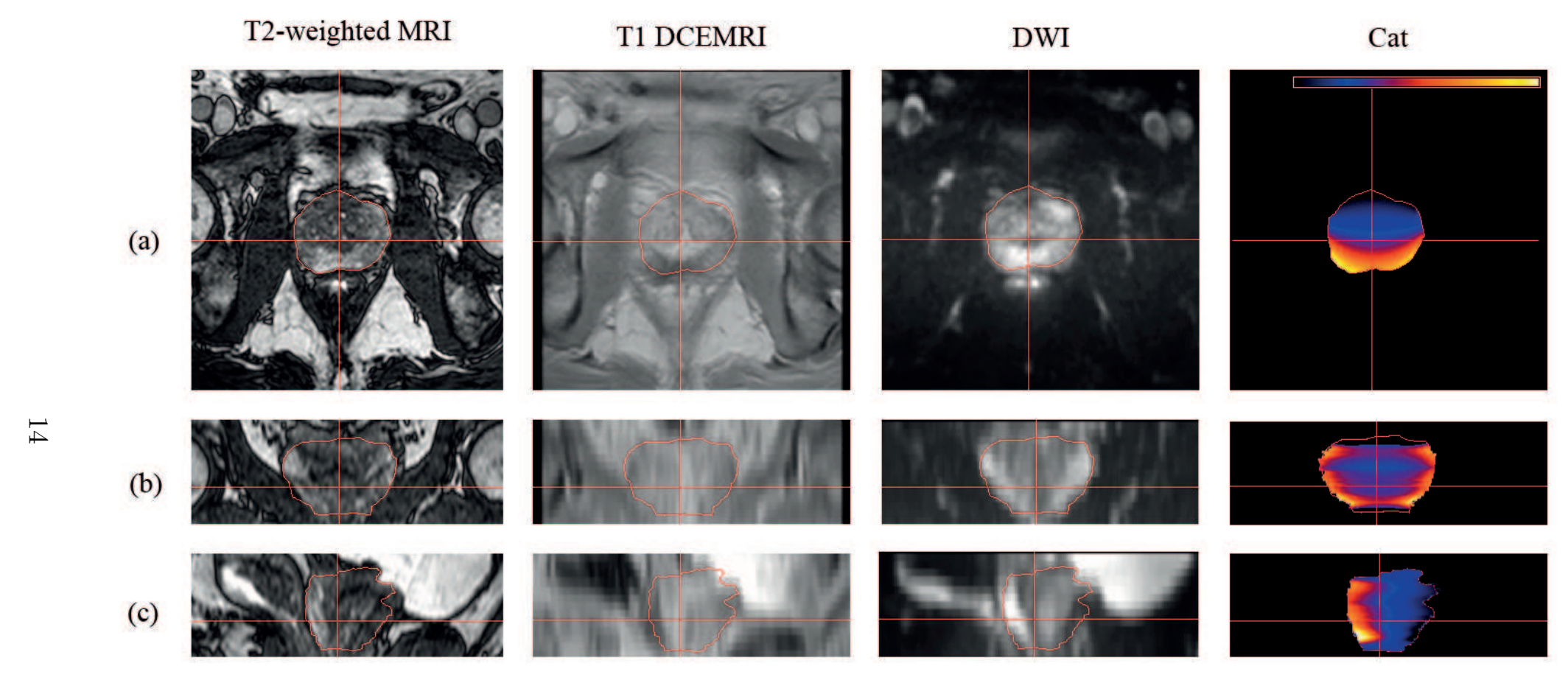

Figure 3: Illustration of multi-source data used for segmentation of prostate zones. Data volumes are viewed in axial (a), coronal (b) and sagital (c) views. From left to right : T2-weighted MRI, T1-weighted Contrast-Enhanced MRI, Diffusion-weighted MRI and Cat-based Morphological a priori feature. Prostate contours are in red. 


\section{Experiments and results}

In this section, ECM refers to the Evidential C-Means [19] and MECM (Modified ECM) refers to our segmentation scheme. These methods were first confronted on simulated images to evaluate the improvements brought by our scheme, MECM, in a segmentation context. The choice of simulated images was to allow control of image quality, using several contrast and Signal to Noise Ratio values in the rendering process. Second, our scheme was tested on real patients MRI data, to evaluate its performances and feasibility in a clinical context. Default values were used for ECM's and MECM's shared parameters : $a=2, b=2$ and $\delta=\sqrt{20}$. Optimal value of MECM's parameter $\gamma$ had been estimated by the experiment detailed in paragraph 5.2. Conjunctive non-normalised combination rule was used when applying MECM but, as mentioned in paragraph 3.3.1, the impact of this choice is discussed in section 6.1.

\subsection{Data}

\subsubsection{Simulated images}

We simulated multi-source images using real prostate MRI series, contoured by an expert radiologist. The purpose of this operation is to render image objects that are shaped like real prostate structures (PZ and TZ). Predelineated $\mathrm{TZ}$ was filled with its mean $\mathrm{MR}$ value. The PZ level is deduced using a pre-determined contrast value, given by

$$
C=\frac{\left|\mathrm{I}_{T Z}-I_{P Z}\right|}{\mathrm{I}_{T Z}+I_{P Z}}
$$

where $I_{T Z}$ and $I_{P Z}$ are mean MR signals of TZ and PZ. We add Gaussian noise, with a chosen standard deviation, and we apply standard median filter to reduce salt-pepper aspect of the noisy images. The same process was used to render images from T2-w, DWI and CE MRI. Figure 4 illustrates this process. We define Signal-to-Noise Ratio of this simulated data as:

$$
S N R_{d b}=10 \log \left[\frac{\sum_{i=1 \ldots n} I_{0}\left(v_{i}\right)^{2}}{\sum_{i=1 \ldots n}\left(I\left(v_{i}\right)-I_{0}\left(v_{i}\right)\right)^{2}}\right]
$$

where $I_{0}\left(v_{i}\right)$ and $I\left(v_{i}\right)$ are intensities of voxel $v_{i}$ before and after adding the Gaussian noise, respectively. 

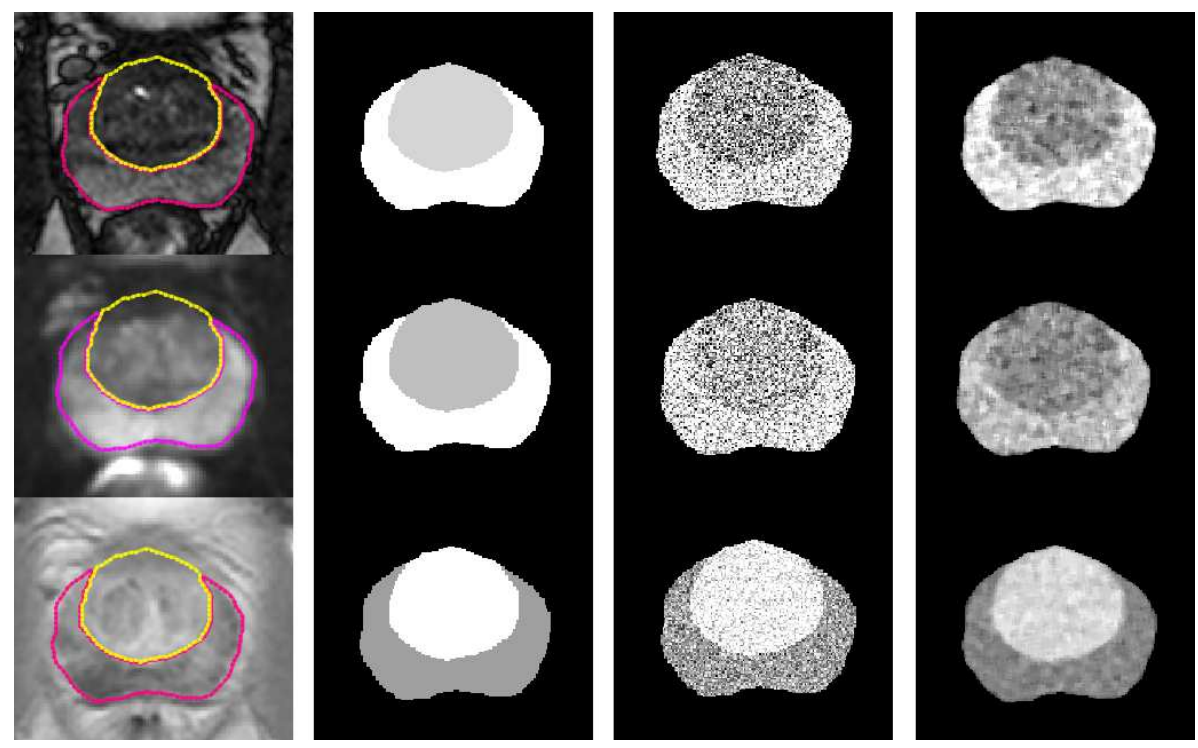

Figure 4: Image simulation process. From Left to right : original MRI with pre-delineated PZ and TZ, PZ and TZ labelled with their respective mean level, data with Gaussian noise, data after a smoothing median filter. First, second and third lines represent T2-weighted, T1-CEMRI and DWI, respectively.

\subsubsection{Real MRI data}

Patients MR images were provided by radiology department of Claude Huriez hospital. Acquisitions were made on a 1.5T Philips MRI device. 31 patients exams were used in our experiments. These exams include complete multi-parametric MRI and cancer diagnosis data. 18 patients $(56.25 \%$ of the group) had been diagnosed "positive" for prostate cancer. Three MRI series were used in this study : T2-weighted with $0.48 \times 0.48 \times 1.25 \mathrm{~mm}$ voxel size, T1 CEMRI with $0.62 \times 0.62 \times 4.00 \mathrm{~mm}$ voxel size, and DWI with $1.12 \times 1.12 \times 4.00 \mathrm{~mm}$ voxel size. These MR volumes were rescaled to fit the highest resolution $(0.48 \times 0.48 \times 1.25 \mathrm{~mm})$ in order to avoid data loss. All features, including MRI and morphological a priori, were linearly normalised to the same range of values. The latter was automatically defined as the widest range among data channels.

\subsection{Optimal weighting parameter}

We estimate the optimal value of parameter $\gamma$ defined in equation 9 . An optimal value of $\gamma$ is crucial in the process of introducing spatial neighbourhood information, and should not be correlated to specific image situations/conditions. 
We tested the MECM method on simulated images with different values of $\gamma$, and several contrast $(0.15 \leq C \leq 0.3)$ and Signal to Noise Ratios $(8 d B \leq S N R \leq 16 d B)$. These contrast/noise levels were chosen regarding real prostate MRI contrast/noise. SNRs and contrasts (mean \pm std.dev) of the MRI used in this study (T2-w, T1 CEMRI and DWI) were of $10.8 \pm$ $1,5 \mathrm{~dB}$ and $0.272 \pm 0.037$, respectively. Figure 5 shows MECM's classification error (mean \pm std.dev) for $0 \leq \gamma \leq 1$. The classification error is defined as the ratio of the number of misclassified voxels (compared to ground truth) to the total number of voxels.

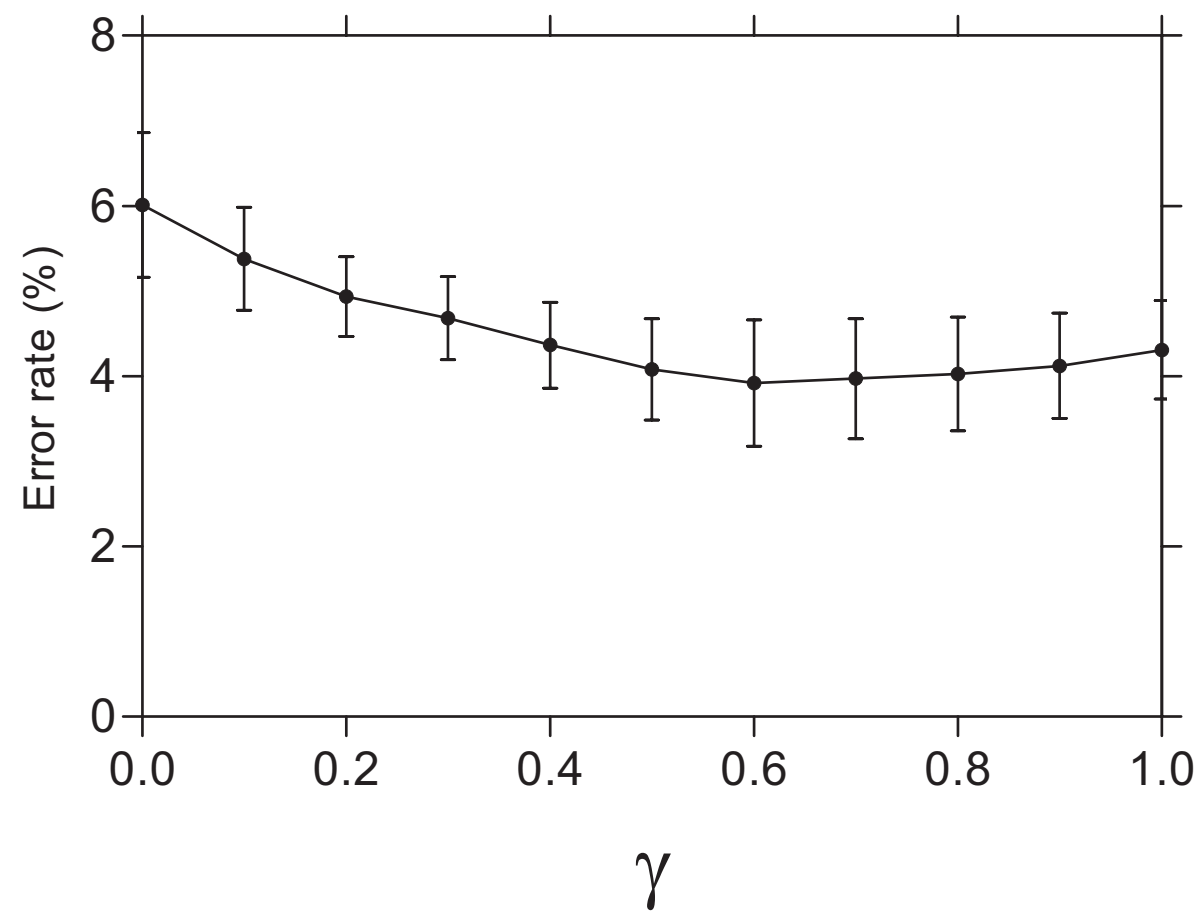

Figure 5: Experimental estimation of $\gamma$ 's optimal value : MECM classification errors $($ mean \pm std.dev $)$ for several contrast and noise levels.

We could establish that classification errors have global minima for $0.5 \leq$ $\gamma \leq 0.7$. For the following experiments, we choose $\gamma=0.6$.

\subsection{Impact of contextual information : MECM Vs ECM}

In this experiment, we assess the introduction of spatial neighbourhood information. Simulated data volumes were rendered using the process described in section 5.1.1. Rendered images had different quality levels: Signal 
to Noise Ratios were from $18.21 d B$ to $7.77 d B$, and contrast levels were from 0.1 to 0.25 . We compare segmentation results from ECM and MECM to ground truth. Segmentation accuracy is measured by Dice similarity coefficient (DSC), Overlap Ratio (OR, also called Jaccard Index), and Volume Difference Ratios (VDR). We also measured the accuracy of manual segmentation. Results of this study are detailed in table 2, and a visual comparison of classification results is given in figure 6 .

\begin{tabular}{cccccccc} 
& & \multicolumn{2}{c}{ DSC } & \multicolumn{2}{c}{ OR } & \multicolumn{2}{c}{ VDR } \\
& & Mean & Std. dev. & Mean & Std. dev. & Mean & Std. dev. \\
\hline \multirow{2}{*}{ ECM } & PZ & 0.6711 & 0.1594 & 0.5159 & 0.1819 & -0.7605 & 0.6382 \\
& TZ & 0.8383 & 0.0501 & 0.7232 & 0.0744 & 0.2006 & 0.1038 \\
\hline \multirow{2}{*}{ MECM } & PZ & 0.8524 & 0.0260 & 0.7433 & 0.0395 & -0.1246 & 0.0732 \\
& TZ & 0.9418 & 0.0331 & 0.8910 & 0.0592 & 0.0145 & 0.0730 \\
\hline \multirow{2}{*}{ Manual } & PZ & 0.9256 & 0.0286 & 0.8622 & 0.0495 & -0.0481 & 0.0241 \\
& TZ & 0.9755 & 0.0008 & 0.9523 & 0.0016 & 0.0080 & 0.0033 \\
\hline
\end{tabular}

Table 2: Results on simulated datasets : ECM Vs MECM Vs manual segmentation. 

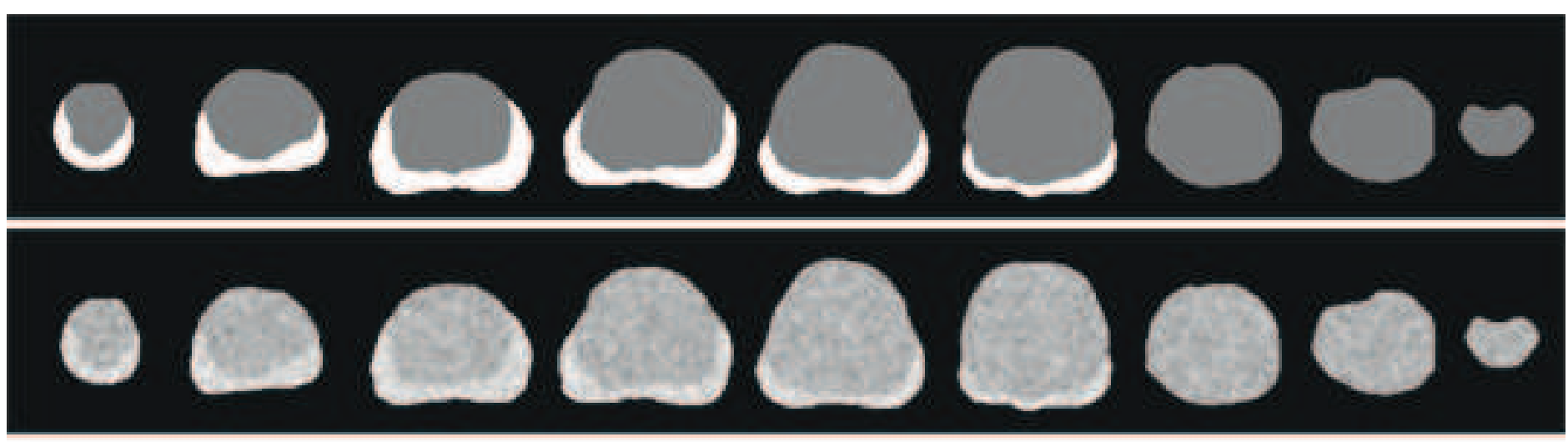

$\breve{\bullet}$
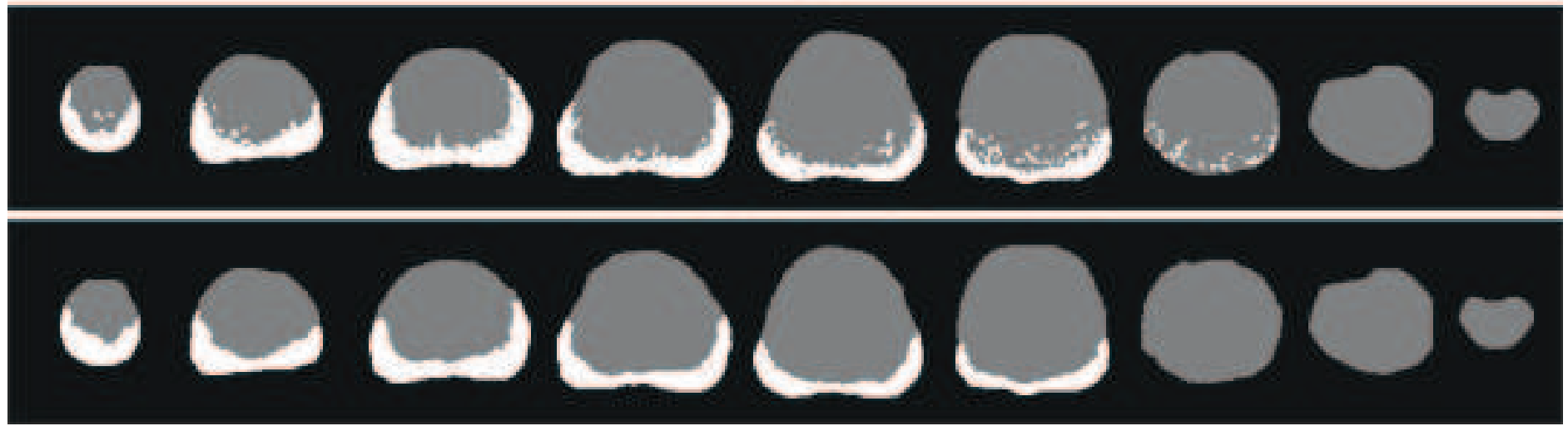

Figure 6: Illustration of the impact of introducing contextual information and morphological feature: segmentation results from ECM and MECM (third and fourth rows from the top, respectively) are viewed in axial slices, from prostate's apex (left) to the base (right). TZ and PZ are labelled grey and white, respectively. First and second rows represent ground truth and simulated noisy data, respectively. 
With a DSC of $0.941 \pm 0.033$ for TZ (0.852 \pm 0.026 for PZ), segmentation from MECM - which introduces contextual neighbourhood information - was satisfyingly close to expert segmentation, which DSC was of $0.975 \pm 0.001$ for TZ $(0.925 \pm 0.028$ for PZ). The MECM proved to be more accurate than ECM, which is due to the introduction of contextual neighbourhood information. The latter allows the algorithm acting like a region-based segmentation method. Visual results from MECM segmentation (fourth row), reveal two connected regions $\mathrm{PZ}$ and $\mathrm{TZ}$, in which the number of misclassified voxels was significantly reduced compared to ECM classification. The modelling of spatial connexity allowed to correct the belief on voxels' membership; outicantly reduces labelling errors. Overlap Ratios (mean \pm std.dev) confirm these observations, with $0.723 \pm 0.074$ and $0,891 \pm 0.059$ for TZ segmentation from ECM and MECM, respectively. Expert manual segmentation of $\mathrm{TZ}$ had an OR of $0.952 \pm 0.001$. As a global measure of MECM's impact,

370 figure 7 shows that the method is much less sensitive to noise than ECM; MECM labelling errors were below $5 \%$ even for extremely noisy data (eg. for $S N R=7.77 d B$ ), while errors from ECM reached $20 \%$.

\subsection{Results on patients $M R I$}

31 patients multispectral MRI (described in section 4.1.2) were used to validate and assess the performance of the proposed MECM method. In order to study the impact of inter-observer variability, the images were contoured by three experts (Obs1, Obs2 and Obs3) from radiology department of Claude-Huriez's Hospital. Each patient MR exam could then be associated to three independent and different segmentations. Based on these segmentations, we computed a consensual expert segmentation from the observers' labellings using "Simultaneous Truth And Performance Level Estimation" algorithm (STAPLE) [27]. This algorithm allows to estimate the hidden ground truth from a collection of different labellings of the same object using an expectation-maximization of each labelling performance. We then compared automatic segmentation, expert segmentation and the estimated STAPLE using DSC, OR, and VDR. Results of these tests are summarised in table 3 .

Computation time required by MECM, on a standard Personal Computer (AMD AthlonXP CPU, 2 Gigabytes of RAM), was of $9 \pm 2 \mathrm{~min}$, while

it took the radiologist $32 \pm 9 \mathrm{~min}(($ mean \pm std.dev $)$ to delineate prostate $1.25 \mathrm{~mm}$-thick T2-weighted MR Images.

Compared to STAPLE estimated truth, MECM segmentation had a median DSC of 0.88 and 0.78 , for $\mathrm{TZ}$ and PZ, respectively. Interquartile ranges 

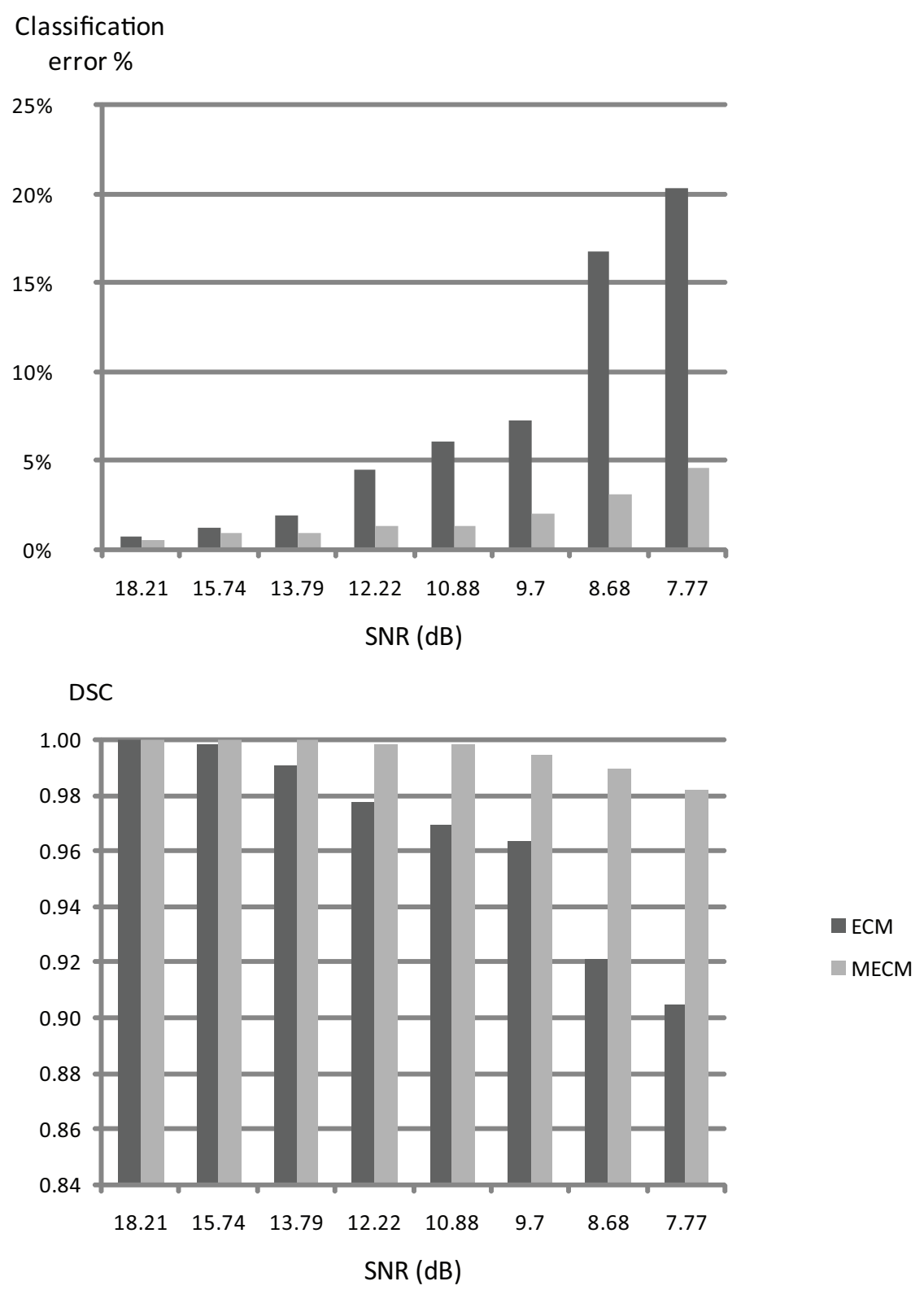

Figure 7: MECM Vs ECM: Mean errors rate and DSC from ECM and MECM results on Simulated data with different SNRs.

of MECM DSCs were of $[0.84,0.90]$ for TZ and $[0.70,0.81]$ for PZ, which 395 means that for all patients MRI included in these tests, the MECM seg- 


\begin{tabular}{|c|c|c|c|c|c|c|c|}
\hline \multirow[b]{2}{*}{$M E C M$ versus } & & \multicolumn{2}{|c|}{ DSC } & \multicolumn{2}{|c|}{ OR } & \multicolumn{2}{|c|}{ VDR } \\
\hline & & Median & IQR & Median & IQR & Median & IQR \\
\hline \multirow{2}{*}{ Obs1 } & $\overline{\mathrm{PZ}}$ & 0.71 & {$\left[\begin{array}{ll}0.65 & 0.78\end{array}\right]$} & 0.56 & {$\left[\begin{array}{ll}0.49 & 0.68\end{array}\right]$} & -0.2 & {$\left[\begin{array}{ll}-0.31 & -0.12\end{array}\right]$} \\
\hline & $\mathrm{TZ}$ & 0.82 & {$\left[\begin{array}{ll}0.78 & 0.86\end{array}\right]$} & 0.70 & {$\left[\begin{array}{ll}0.64 & 0.75\end{array}\right]$} & 0.17 & {$\left[\begin{array}{ll}0.05 & 0.37\end{array}\right]$} \\
\hline \multirow{2}{*}{ Obs2 } & $\overline{\mathrm{PZ}}$ & 0.78 & {$\left[\begin{array}{lll}0.69 & 0.81\end{array}\right]$} & 0.63 & {$\left[\begin{array}{lll}0.52 & 0.68\end{array}\right]$} & -0.12 & {$\left[\begin{array}{lll}-0.18 & 0.03\end{array}\right]$} \\
\hline & $\mathrm{TZ}$ & 0.89 & {$\left[\begin{array}{ll}0.86 & 0.93\end{array}\right]$} & 0.80 & {$\left[\begin{array}{ll}0.76 & 0.87\end{array}\right]$} & 0.05 & {$\left[\begin{array}{ll}0 & 0.12\end{array}\right]$} \\
\hline \multirow{2}{*}{ Obs3 } & $\overline{\mathrm{PZ}}$ & 0.7 & {$\left[\begin{array}{ll}0.62 & 0.78\end{array}\right]$} & 0.54 & {$\left[\begin{array}{ll}0.51 & 0.64\end{array}\right]$} & -0.06 & {$\left[\begin{array}{ll}-0.15 & 0.05\end{array}\right]$} \\
\hline & $\mathrm{TZ}$ & 0.81 & {$\left[\begin{array}{ll}0.77 & 0.87\end{array}\right]$} & 0.69 & {$\left[\begin{array}{ll}0.62 & 0.78\end{array}\right]$} & 0.08 & {$\left[\begin{array}{ll}0.01 & 0.13\end{array}\right]$} \\
\hline \multirow{2}{*}{ STAPLE } & $\mathrm{PZ}$ & 0.78 & {$\left[\begin{array}{ll}0.69 & 0.81\end{array}\right]$} & 0.63 & {$\left[\begin{array}{ll}0.54 & 0.67\end{array}\right]$} & -0.11 & {$\left[\begin{array}{ll}-0.16 & 0.0\end{array}\right]$} \\
\hline & $\mathrm{TZ}$ & 0.88 & {$\left[\begin{array}{ll}0.83 & 0.90\end{array}\right]$} & 0.78 & {$\left[\begin{array}{ll}0.71 & 0.81\end{array}\right]$} & 0.14 & {$\left[\begin{array}{ll}0.04 & 0.16\end{array}\right]$} \\
\hline \multicolumn{8}{|l|}{ ECM versus } \\
\hline \multirow{2}{*}{ Obs1 } & $\overline{\mathrm{PZ}}$ & 0.62 & {$\left[\begin{array}{ll}0.47 & 0.75\end{array}\right]$} & 0.45 & {$\left[\begin{array}{ll}0.31 & 0.6\end{array}\right]$} & -0.14 & {$\left[\begin{array}{ll}-0.21 & 0.91\end{array}\right]$} \\
\hline & $\mathrm{TZ}$ & 0.78 & {$\left[\begin{array}{ll}0.7 & 0.81\end{array}\right]$} & 0.71 & {$\left[\begin{array}{ll}0.59 & 0.74\end{array}\right]$} & 0.07 & {$\left[\begin{array}{ll}-0.16 & 0.19\end{array}\right]$} \\
\hline \multirow{2}{*}{ Obs2 } & $\overline{\mathrm{PZ}}$ & 0.62 & {$\left[\begin{array}{lll}0.46 & 0.74\end{array}\right]$} & 0.46 & {$\left[\begin{array}{ll}0.29 & 0.58\end{array}\right]$} & 0 & {$\left[\begin{array}{ll}-0.18 & 0.76\end{array}\right]$} \\
\hline & $\mathrm{TZ}$ & 0.76 & {$\left[\begin{array}{ll}0.69 & 0.8\end{array}\right]$} & 0.62 & {$\left[\begin{array}{ll}0.52 & 0.67\end{array}\right]$} & 0.07 & {$\left[\begin{array}{ll}-0.1 & 0.23\end{array}\right]$} \\
\hline \multirow{2}{*}{ Obs3 } & $\mathrm{PZ}$ & 0.61 & {$\left[\begin{array}{ll}0.44 & 0.67\end{array}\right]$} & 0.43 & {$\left[\begin{array}{ll}0.29 & 0.5\end{array}\right]$} & -0.21 & {$\left[\begin{array}{ll}-0.35 & 0.84\end{array}\right]$} \\
\hline & $\mathrm{TZ}$ & 0.74 & {$\left[\begin{array}{ll}0.65 & 0.79\end{array}\right]$} & 0.59 & {$\left[\begin{array}{ll}0.48 & 0.65\end{array}\right]$} & 0.15 & {$\left[\begin{array}{ll}-0.13 & 0.75\end{array}\right]$} \\
\hline \multirow{2}{*}{ STAPLE } & $\overline{\mathrm{PZ}}$ & 0.66 & {$\left[\begin{array}{lll}0.47 & 0.75\end{array}\right]$} & 0.48 & {$\left[\begin{array}{ll}0.31 & 0.6\end{array}\right]$} & -0.14 & {$\left[\begin{array}{ll}-0.23 & 0.87\end{array}\right]$} \\
\hline & $\mathrm{TZ}$ & 0.79 & {$\left[\begin{array}{ll}0.73 & 0.82\end{array}\right]$} & 0.66 & {$\left[\begin{array}{ll}0.57 & 0.7\end{array}\right]$} & -0.14 & {$\left[\begin{array}{ll}-0.23 & 0.87\end{array}\right]$} \\
\hline
\end{tabular}

Table 3: Performances (medians and InterQuartile Ranges) of ECM and MECM tested on MRI data from 31 patients. Both ECM and MECM are compared to 3 expert observers (Obs1, Obs2 and Obs3) and the estimated truth (STAPLE).

mentation was satisfyingly close to human observers. The quality of MECM segmentation is visualised on figure 8; the extracted PZ seems very similar to both expert segmentation and the estimated truth. The method specifically succeeded in dealing with signal overlap from different structures; we notice that the presence of cysts (with PZ's image level) did not induce segmentation errors (figure 8). We notice also that the apex and base slices (left and right columns, respectively), were segmented successfully despite the critical homogeneity and lack of contrast in these parts. These results also prove that MECM outperforms ECM when tested on real data. This is shown by DSC, OR and VDR values of Table 3, and is illustrated by PZ labels of figure 8. MECM segmentation particularly succeeds in avoiding outliers and providing a well-connected region that represents PZ, while ECM remains sensitive to local variations of intensities and groups prostate voxels that share intensities, regardless of their spatial context.

We also tested the sensitivity of our method and the expert observers to 


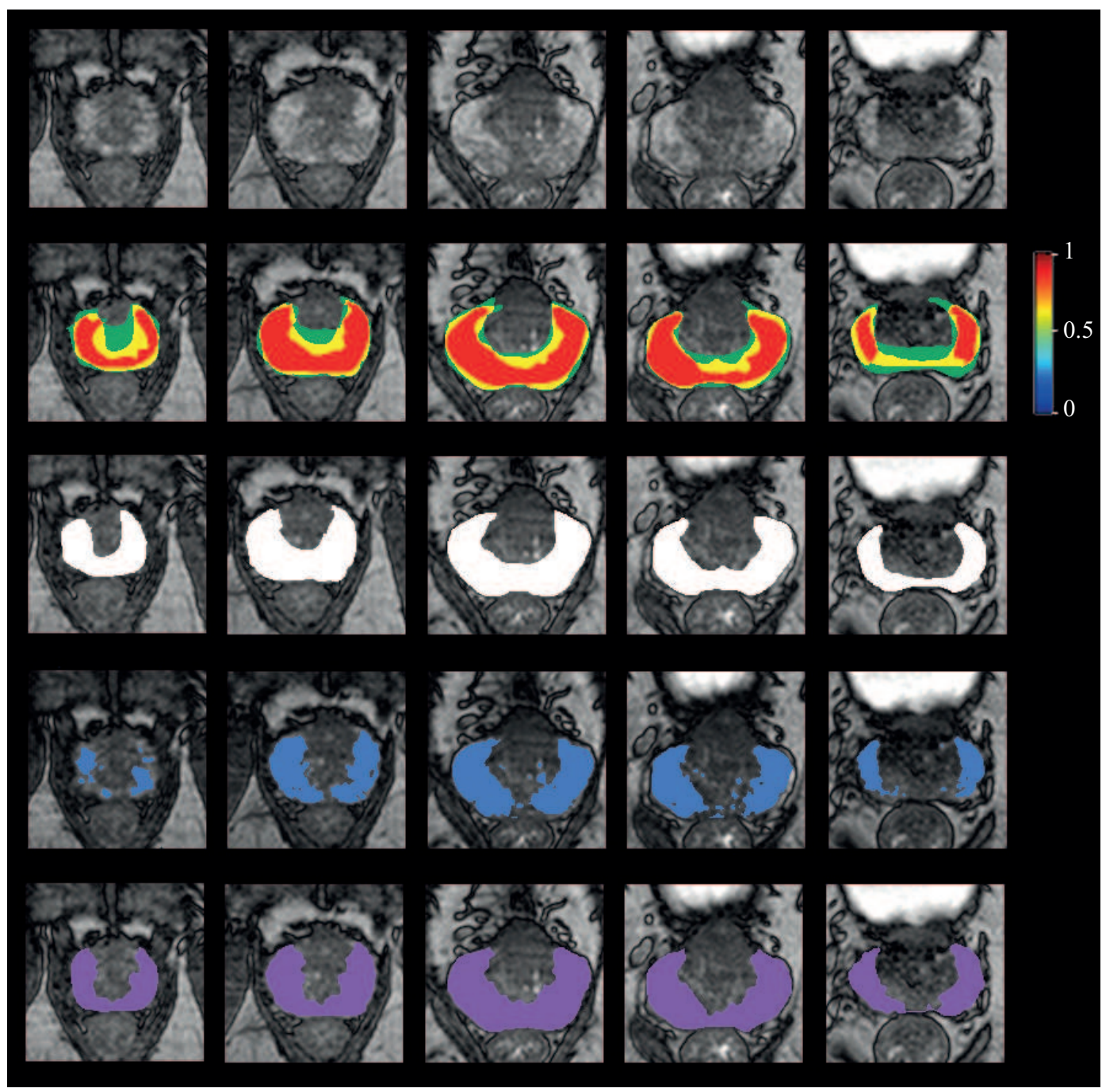

Figure 8: An illustration of PZ segmentation from MECM (bottom row, PZ is labelled in purple) and ECM (PZ is labelled in blue) compared to STAPLE's estimated truth (third row from the top, PZ is labelled in white). Differences between segmentations from expert observers, (Obs1, Obs2 and Obs3), are highlighted by a map of labelling frequency, as shown in the second row. All labellings are presented on axial views from T2-weighted MRI (top row).

the presence of tumours and to variations in prostate shape and appearance. Tumours, age and prostate volume are major factors that affect prostate tissues appearance on MRI studies. We partitioned the test population into 
groups, based on three criteria: age, prostate volume, and cancer diagnosis.

415 We used Mann-Whitney's non-parametric t-test to assess, for each criterion, if segmentation accuracy were different for each group. For each criterion, the NULL hypothesis was then: The mean DSC of the segmentation method for group 1 is equal to the mean DSC for group 2. A p-value that is superior to 0.05 would mean, with type1 error of $5 \%$, that the NULL hypothesis was true. In other word, the highest the p-value, the less sensitive the segmentation method to the criterion. Table 4 summarises p-values for MECM and the three experts. These p-values show that results from MECM were not significantly different for the groups of patients. The MECM proved even to be much less sensitive to the presence of tumors than experts Obs1 and Obs2.

\begin{tabular}{cccccc} 
& & \multicolumn{4}{c}{ Method } \\
Criterion & & MECM & Obs1 & Obs2 & Obs3 \\
\hline \multirow{2}{*}{ Patient's age } & TZ & 0.8857 & 0.8849 & 0.5614 & 0.9800 \\
& PZ & 0.8857 & 0.2000 & 0.1143 & 0.8857 \\
Prostate's volume & TZ & 0.5714 & 0.3826 & 0.7857 & 0.9800 \\
& PZ & 0.3929 & 0.3754 & 0.3929 & 0.5714 \\
Tumour presence & TZ & 0.2000 & 0.3429 & 0.9800 & 0.4857 \\
& PZ & 0.9200 & 0.0286 & 0.0286 & 0.6857 \\
\hline
\end{tabular}

Table 4: P-values of tests of the sensitivity of manual (Obs1, Obs2 and Obs3) and automatic (MECM) segmentation to three different criteria: patient's age, prostate's volume and presence/absence of tumours. The highest the p-value, the less sensitive the segmentation method. $(\mathrm{p}=0.05)$ is the threshold below which results of the segmentation method are significantly different for the two groups, and thus influenced by the criterion.

\section{Discussion}

\subsection{Impact of belief combination rules}

Several combination rules may be used in introducing neighbourhood informations, and we made the choice of using conjunctive rules, normalised and non-normalised (c.f. section 3.3.1). Figure 9 shows a comparison of credal partitions obtained using each one of these rules. 

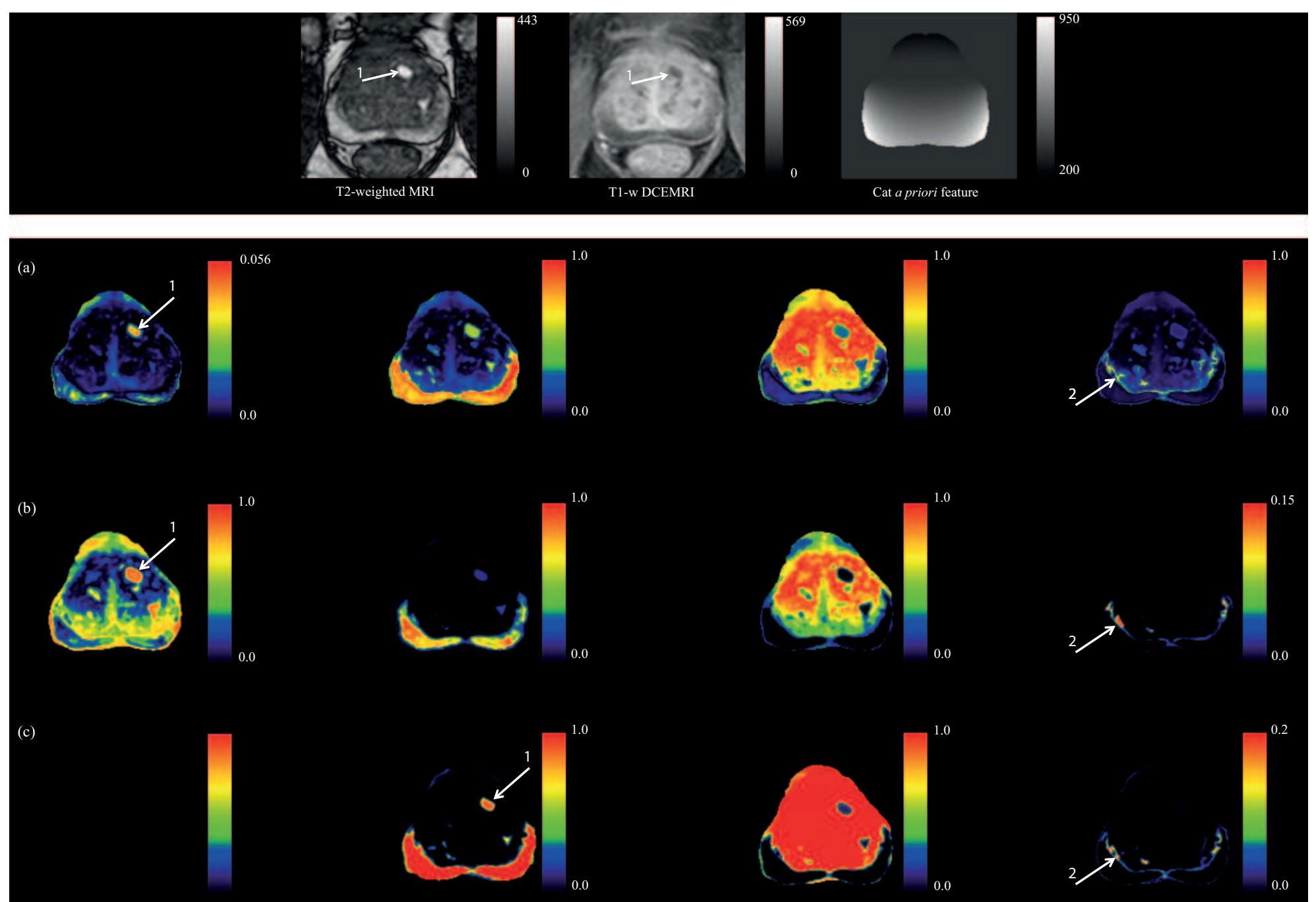

$\mathrm{m}(\varnothing)$

$\mathrm{m}(\mathrm{PZ})$

$\mathrm{m}(\mathrm{TZ})$

$\mathrm{m}(\Omega)$

Figure 9: Maps of bbas obtained with ECM (a), MECM using non-normalised conjunctive combination (b) and using Dempster's normalised conjunctive combination (c). Maps, in colour scale, show (from left to right) $m(\emptyset), m\left(\omega_{T Z}\right), m\left(\omega_{P Z}\right)$ and $(m(\Omega))$. Top row shows data sources (from left to right : T2-w, T1-CEMRI MRI and a priori). White Arrows 1 and 2 indicate a cyst and the frontier between $\mathrm{PZ}$ and the centre of the gland, respectively. 
First, it appears that non-normalised combination allows smoother bbas on borders of regions PZ and TZ, while Dempsters' normalised combination tends to be more deterministic. The use of the latter, which normalises $m(\emptyset)=0$, may cause a loss of valuable information that can be seen on $m(\emptyset)$ 's maps from ECM and the non-normalised rule (figure 9: (a) and (b)). Dempsters rule was proved [28] but also criticized because of its normalisation step [29]. One of the alternatives is to avoid the normalisation step and to interpret the belief assigned to $\emptyset$, sometimes called conflict information. We note that senior radiologists made very interesting analysis of belief maps, which provide more information than a classical binary decision. This analysis and the interpretation of $m(\emptyset)$ and $m(\Omega)$ in the context of MECM's scheme are detailed in the following paragraph.

\subsection{Analysing conflict and ignorance information}

In ECM's classification scheme, $m(\emptyset)$ is modelled and depends on $\delta$ (equations 3 and 6), which can be considered as a threshold on distances to classes' barycentres [19] :

$$
\delta^{2}=\lambda \frac{1}{N . C}\left(\sum_{i=1}^{N} \sum_{j=1}^{C} d_{i j}^{2}\right)
$$

where $\lambda$ is a weighting parameter (default value $=\sqrt{20}$ ).

As shown by Figure 9, there is a region of high $m(\emptyset)$ values within the centre of the gland, shown by white arrow 1 . The intensities from this region are similar to ones from PZ (c.f. first row of figure 9). According to expert radiologist's analysis, this region represents a cyst of the Transition Zone. According to belief maps, voxels from this region had been assigned to the empty set by ECM and MECM using non-normalised conjunction (rows (a) and (b)). The MECM using normalised conjunction assigned these voxels to PZ (row (c)). These cysts are not modelled in the frame of discernment $\Omega$, and were assigned, as outliers, to the empty set $\emptyset$. To be less specific, when using the proposed segmentation scheme with non-normalised combination rule, bbas assigned to the empty set can be interpreted as outliers and structures that were not modelled in the frame of discernment. In this case, a part of $m(\emptyset)$ can be used to detect one or several hypotheses that have not been initially taken into account. In addition, non-normalised conjunctive combination (equation 8) generates additional levels of $m(\emptyset)$ induced by possible discordance - conflict - between neighbour bbas. Figure 9.b shows amount of conflict information located on frontiers between the two classes, PZ and TZ, where voxels of the same neighbourhood belong 
to different structures and then bring conflictual information. $m(\emptyset)$ maps may then represent both regions' borders and outliers. Even though there is no unique interpretation of $m(\emptyset)$ maps, it can be helpful for guiding human 470 expert analysis and decision, by highlighting irregularities and "unexpected" structures. Less specifically, it can be used to model rejection of outliers or unwanted noise regions in the segmentation scheme.

On the other hand, we also notice high levels of $m(\Omega)$ near regions' frontiers, where voxels are likely to belong to one of the two structures.

475 These levels of belief draw a frontier between the two regions (PZ and TZ) and, when compared to image gradient (figure 10), it appears that $m(\Omega)$, especially those computed using MECM, are more specific to the forntier between the regions defined in the frame of discernment. Such an information can be used in edge detection techniques such as active contours/surfaces in which contours computation methods (like image gradient and Gradient Vector Flow) have serious issues dealing with borders between regions that are not targeted by the segmentation process.

\subsection{Conclusion}

In this paper, we introduced a novel evidential segmentation scheme for multi-source images and presented its application to multi-parametric prostate MRI. This method is based on the Evidential C-Means classifier in which we introduced a relaxation step to integrate voxels' spatial neighbourhood information. The choice of using belief functions through the ECM model was motivated by the ability of this theory to take into account incomplete, redundant and complementary data sources, which are properties of prostate multi-parametric MRI. Belief functions modelling also offers a set of native rules for data fusion and belief combination which we used to model the partial knowledge brought by connected neighbours on voxels membership. The use of belief combination and weakening allowed us to model the assumption of spatial connexity of similar regions, and to adapt the ECM classifier to image segmentation process. The impact of this technique was shown by quantitative and qualitative enhancement in segmentation quality. We have shown that the modified ECM scheme, MECM, modifies the final decision and provides a more accurate segmentation: this new process leads to a true region-based segmentation scheme, providing better connected regions with smoother boundaries. Via neighbourhood information, the MECM also succeeded in classifying noisy voxels and outliers, and the remaining ambiguities are mainly located on the frontiers between regions. We also tested our MECM segmentation scheme on 31 patients prostate multi-parametric MRI exams, and compared its performance based on three 


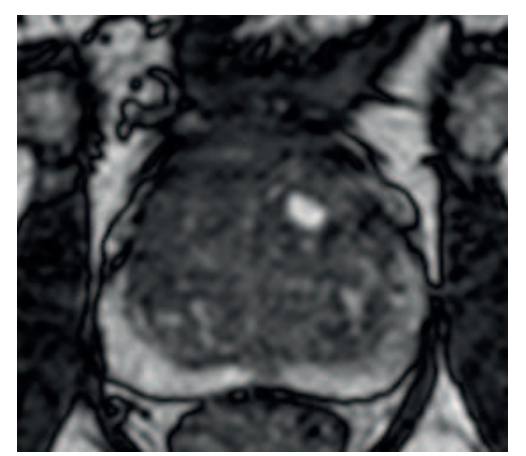

(a)

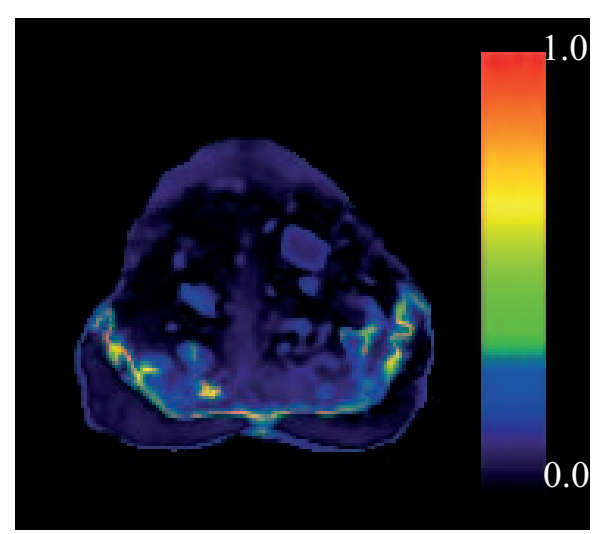

(c)

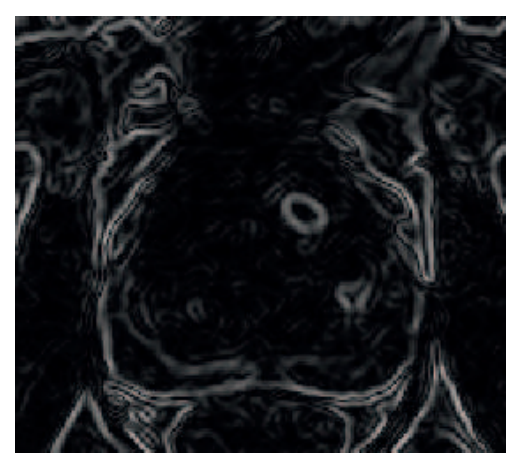

(b)

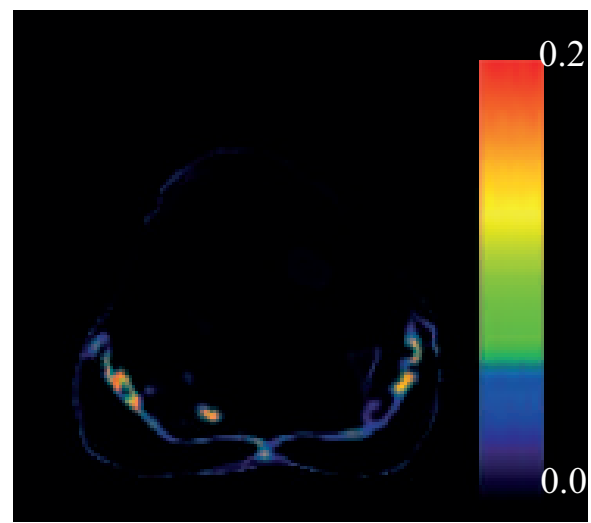

(d)

Figure 10: Edge extraction using basic belief assignment to $\Omega$. Maps of $m(\Omega)$ are shown for ECM (c) and MECM (d). First row shows, from left to right, T2-W MRI and the associated gradient image.

different and independent experts in order to take into account the interobserver variability. MECM segmentation results were satisfyingly accurate and similar to expert truth, estimated using STAPLE multi-observer truth estimator. Our method succeeded in avoiding ambiguous signals, e.g. PZlike signals from cysts in the TZ, and provided two well connected regions representing the two zones. This paper brings then a novel method that allows using the approximate Evidential reasoning in segmentation applications. The scope of this paper was not to compare its performance to other generic stae-of-the art segmentation algorithms, but to prove its feasibility in the context of belief functions theory. We are currently working on such a comparative study which will be the subject of a dedicated paper. 
Nevertheless, in order to give the reader a basic idea on the effectiveness of MECM, we show in figure 11 a comparison to segmentation based on Gaussian Mixture Models (GMM). On both simulated and real multispectral prostate MR data, the MECM segmentation is visually but significantly better than ECM segmentation, and the latter is slightly better than GMM. A full comparison of MECM is to be expected in our upcoming paper.

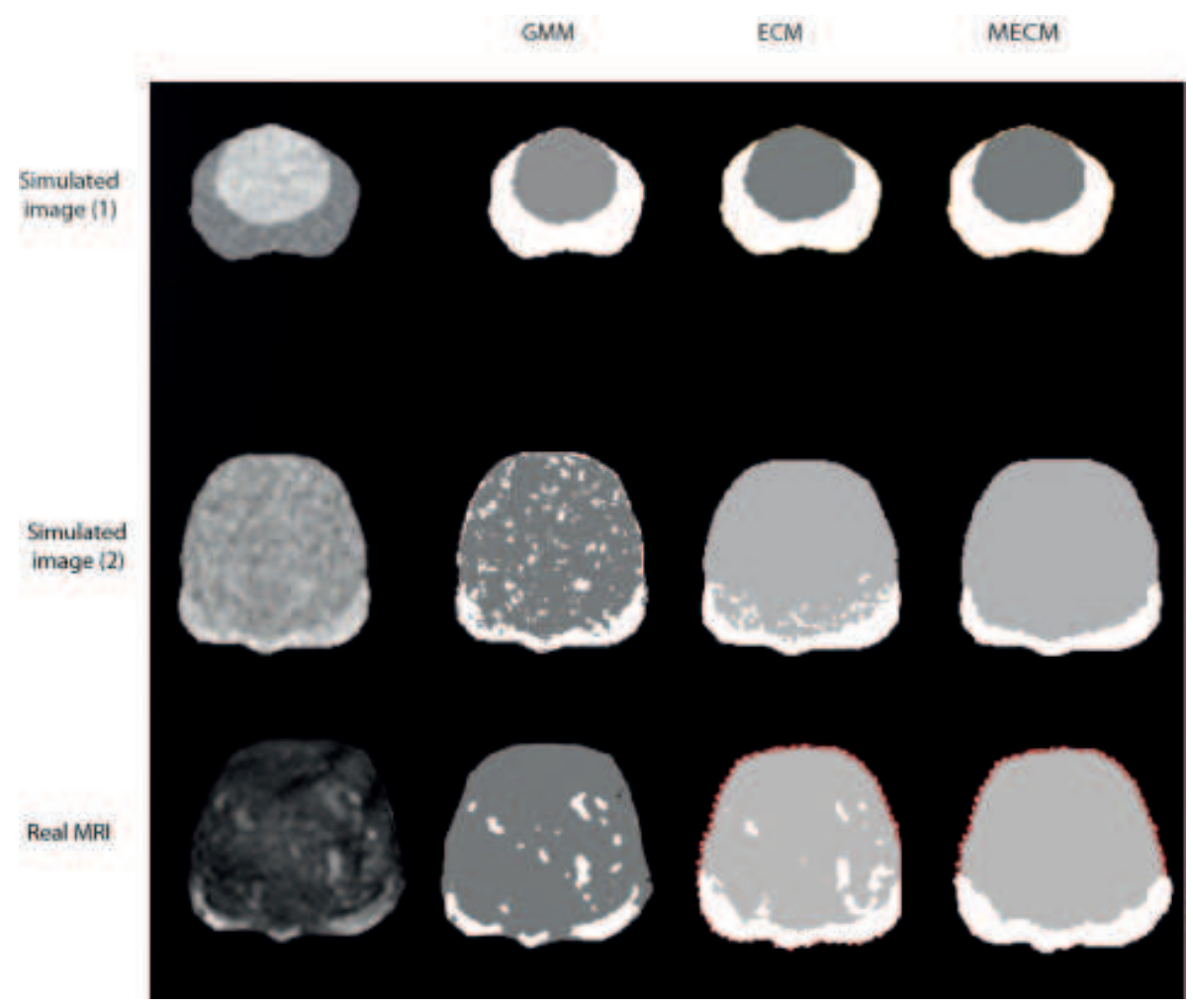

Figure 11: Comparison of ECM and MECM segmentation to Gaussian Mixture Modelbased segmentation (GMM). Simulated image (1) has higher contrast than simulated image (2).

Some limitations of the method could be identified. First one is the use of the neighbourhood-based relaxation as an independent step, and not as an additional criterion in ECM's objective function. The impact of this limitation is basically that the convergence of our algorithm is not based a neighbourhood-aware constraint, but is altered by an additional step which succeeded as proven by experiments. We are looking forward to investigate this point by integrating spatial neighbourhood in a new objective function and evaluate the impact of this new convergence. The second 
limit is inherent to the use of evidential reasoning in multi-label classification/segmentation: modelling belief on power sets may result in exponential complexity of operations (combination, weakening, etc.) made on bbas with respect to the number of classes. This issue has been raised by Denoeux and 535 Masson in a very recent paper [30], and their approach may make the MECM more feasible for segmenting more than two regions of the images. Finally, the lack of modelling of the reliabilities of data sources which are equally used to extract knowledge. It would be relevant to quantify, separately, the contribution of each source to enhance bbas extraction. We notice that in 540 the application presented in this study, which is prostate multi-parametric MRI, such a quantification is only possible through investigation in correlations between prostate MRI and histological studies, which is a work in progress in our team. Another approach is also being investigated and consists in applying ECM-based segmentation on each source and combine the resulting bbas to obtain a final decision on voxel's membership. The latter approach needs to be compared to the method introduced in this paper to assess if any improvement in segmentation's quality is possible. 
References

[1] M. S. Atkins, M. S. Drew, Z. Tauber, Towards automatic segmentation of $\mathrm{ms}$ lesions in $\mathrm{pd} / \mathrm{t} 2 \mathrm{mr}$ images, anglais (2000).

[2] A.-S. Capelle, O. Colot, C. Fernandez-Maloigne, Evidential segmentation scheme of multi-echo mr images for the detection of brain tumors using neighborhood information, Information Fusion 5 (3) (2004) 203 -216 .

555 [3] R. H. Lee, R. M. Leahy, Multispectral tissue classification of mr images using sensor fusion approaches, Medical Imaging IV: Image Processing 1233 (1) (1990) 149-157.

[4] B. Flach, E. Kask, D. Schlesinger, A. Skulish, Unifying registration and segmentation for multi-sensor images, Pattern Recognition (2002) 190-197.

[5] J. Chun, I. Greenshields, Classification algorithm for multi-echo magnetic resonance image using gibbs distributions, Image Analysis Applications and Computer Graphics (1995) 419-426.

[6] S. Bricq, C. Collet, J. Armspach, Unifying framework for multimodal brain mri segmentation based on hidden markov chains, Medical Image Analysis 12 (6) (2008) $639-652$.

[7] G. Shafer, A mathematical theory of evidence., Princeton University Press, N.J., 1976.

[8] A. Dempster, Upper and lower probabilities induced by multivalued mapping, Annals of Mathematical Statistics 38 (1967) 325-339.

[9] A. Appriou, Multisensor signal processing in the framework of the theory of evidence, Lecture Series 216 on Applicationof Mathematical Signal Processing Techniques to MissionSystems (1999) 5-31.

[10] L. Fouque, A. Appriou, W. Pieczynski, An evidential markovian model for data fusion and unsupervised image classification, in: Information Fusion, 2000.FUSION 2000.Proceedings of the Third International Conference on, Vol. 1, 2000, pp. TUB4/25-TUB4/32.

[11] A. Bendjebbour, Y. Delignon, L. Fouque, V. Samson, W. Pieczynski, Multisensor image segmentation using dempster-shafer fusion in markov fields context, in: Geoscience and Remote Sensing, IEEE Transactions on, Vol. 39, 2008, pp. 1789-1798. 
[12] A.Taleb-Ahmed, L.Gautier, On information fusion to improve segmentation of mri sequences, Information Fusion 3 (2) (2002) 103-117.

[13] J.-W. Lee, I.-S. Kweon, Vehicle segmentation using evidential reasoning, in: Intelligent Robots and Systems, 1997. IROS '97., Proceedings of the 1997 IEEE/RSJ International Conference on, Vol. 2, 1997, pp. $880-885$.

[14] Y. Bi, J. Guan, D. Bell, The combination of multiple classifiers using an evidential reasoning approach, Artificial Intelligence 172 (15) (2008) $1731-1751$.

[15] I. Bloch, Some aspects of dempster-shafer evidence theory for classification of multi-modality medical images taking partial volume effect into account, Pattern Recognition Letters 17 (8) (1996) 905-920.

[16] S.-Y. J. Chen, W.-C. Lin, C.-T. Chen, Evidential reasoning based on dempster-shafer theory and its application to medical image analysis, Vol. 2032, SPIE, 1993, pp. 35-46.

[17] D. Suh, R. Mersereau, R. Eisner, R. Pettigrew, Automatic boundary detection on cardiac magnetic resonance image sequences for four dimensional visualisation of the left ventricle, in: First conference on Visualization in Biomedical Computing, 1990, pp. 149-156.

[18] P. Kozlowski, S. Chang, E. Jones, K. Berean, H. Chen, S. Goldenberg, Combined diffusion-weighted and dynamic contrast-enhanced mri for prostate cancer diagnosis-correlation with biopsy and histopathology, Journal of Magnetic Resonance Imaging 24 (2006) 108-113.

[19] M.-H. Masson, T. Denoeux, Ecm : An evidential version of the fuzzy c-means algorithm., Pattern Recognition 41 (2008) 1384-1397.

[20] P. Smets, R. Kennes, The transferable belief model, Artificial Intelligence 66 (1994) 191-243.

[21] D. Mercier, B. Quost, T. Denoeux, Contextual discounting of belief functions, Symbolic and Quantitative Approaches to Reasoning with Uncertainty (2005) 552-562.

[22] P. Smets, Analyzing the combination of conflicting belief functions, Information Fusion 8 (4) (2007) 387-412. 
[23] P. Smets, Constructing the pignistic probability function in a context of uncertainty, in: Proceedings of the Fifth Annual Conference on Uncertainty in Artificial Intelligence, 1990, pp. 29-40.

[24] R. Dav, Clustering relational data containing noise and outliers, Pattern Recognition Letters 12 (1991) 657-664.

[25] P. Smets, The combination of evidence in the transferable belief model, IEEE Transactions on Pattern Analysis and Machine Intelligence 12 (5) (1990) 447-458.

[26] N. Makni, P. Puech, R. Lopes, A. Dewalle, O. Colot, N. Betrouni, Combining a deformable model and a probabilistic framework for an automatic $3 \mathrm{~d}$ segmentation of prostate on mri, International Journal of Computer Assisted Radiology and Surgery 4 (2) (2009) 181-188.

[27] S. Warfield, K. Zou, W. Wells, Simultaneous truth and performance level estimation (staple): an algorithm for the validation of image segmentation, IEEE TMI 23(7) (2004) 903-921.

[28] D. Dubois, H. Prade, On the unicity of dempster rule of combination, International Journal of Intelligent Systems 1 (2) (1986) 133-142.

[29] P. Smets, Resolving misunderstandings about belief functions, Int. J. Approx. Reasoning 6 (1992) 321-344.

[30] T. Denoeux, M.-H. Masson, Evidential reasoning in large partially ordered sets, Annals of Operations Research (2011) 1-27. 